\title{
OSCILLATION AND NONOSCILLATION THEOREMS FOR A CLASS OF FOURTH ORDER QUASILINEAR GENERALIZED $\alpha$-DIFFERENCE EQUATIONS
}

\section{SRIMANJU ${ }^{1} \&$ S K. KHADAR BABU ${ }^{2}$}

${ }^{I}$ Research Scholar, Department of Mathematics, Vellore Institute of Technology, Vellore, Tamil Nadu, India

${ }^{2}$ Associate Professor, Department of Mathematics, Vellore Institute of Technology, Vellore, Tamil Nadu, India

ABSTRACT
In this paper, we consider certain generalized quasilinear $\alpha$ - difference equations
$\Delta_{\alpha(\ell)}^{2}\left(\left|\Delta_{\alpha(\ell)}^{2} v(k)\right| \gamma-1 \Delta_{\alpha(\ell)}^{2} v(k)\right)+q(k)|v(\sigma(k))| \beta-1 v(\sigma(k))=0$
Where $\gamma, \beta$ are positive constants; ${ }^{q(k)}$ are positive real valued function, $k_{0} \in \mathrm{K}_{0}=\{1,2, \cdots\}$. An oscillation and
nonoscillation theorem of the above equation is obtained.
KEYWORDS: Quasilinear Generalized Difference Equations, Oscillation and Nonoscillation Theorems, Four Order

Received: Nov 16, 2019; Accepted: Dec 07, 2019; Published: Jun 30, 2020; Paper Id.: IJMPERDJUN2020190

\section{INTRODUCTION}

In this paper, we consider certain quasilinear generalized $\alpha-$ difference equations

$$
\Delta_{\alpha(\ell)}^{2}\left(\left|\Delta_{\alpha(\ell)}^{2} v(k)\right| \gamma-1 \Delta_{\alpha(\ell)}^{2} v(k)\right)+q(k)|v(\sigma(k))| \beta-1 v(\sigma(k))=0,
$$

- $\quad \gamma, \beta$ are positive constants;

- $q(k)$ are positive real valued function. $k_{0} \in \mathrm{K}_{0}=\{1,2, \cdots\}$.

- $\sigma(k) \leq k$, and $\lim _{k \rightarrow \infty} \sigma(k)=\infty$

The Equation (1) can also be expressed as

$$
\Delta_{\alpha(\ell)}^{2}\left(\left(\Delta_{\alpha(\ell)}^{2} v(k)\right) \gamma^{*}\right)+q(k \ell+j)\left(v(\sigma(k)) \beta^{*}\right)=0
$$

In terms of the asterisk notation

$$
\xi_{\eta^{*}}=|\xi| \eta \operatorname{sgn} \xi=|\xi| \eta-1 \xi \xi, \xi \in \mathrm{R}, \eta>0 .
$$

It is clear that if $v(k)$ is a eventually positive solution of (2), then $-v(k)$ is a eventually negative solution of (2).

Lemma 1: Assume that $v(k)$ is an eventually positive solution of (2). Then one of the following two cases holds for all sufficiently large $k$ : 
- $\quad \Delta_{\alpha(\ell)} v(k)>0, \Delta_{\alpha(\ell)}^{2} v(k)>0, \Delta_{\ell}\left(\Delta_{\alpha(\ell)}^{2} v(k)\right) \gamma^{*}>0$

- $\quad \Delta_{\alpha(\ell)} v(k)>0, \Delta_{\alpha(\ell)}^{2} v(k)<0, \Delta_{\ell}\left(\Delta_{\alpha(\ell)}^{2} v(k)\right) \gamma^{*}>0$

Proof: From (2), we have $\Delta_{\alpha(\ell)}^{2}\left(\left(\Delta_{\alpha(\ell)}^{2} v(k)\right) \gamma^{\gamma^{*}}\right)<0$ for all large $k$. It follows that $\Delta_{\alpha(\ell)} v(k), \Delta_{\alpha(\ell)}^{2} v(k), \Delta_{\alpha(\ell)}\left(\Delta_{\alpha(\ell)}^{2} v(k)\right) \gamma^{*}$ are eventually monotonic and one-signed.

- If $\Delta_{\alpha(\ell)}\left(\Delta_{\alpha(\ell)}^{2} v(k)\right) \gamma^{*}<0$ eventually. Then combining this with $\Delta_{\alpha(\ell)}^{2}\left(\left(\Delta_{\alpha(\ell)}^{2} v(k)\right) \gamma^{*}\right)<0$, we see $\lim _{k \rightarrow \infty}\left(\Delta_{\alpha(\ell)}^{2} v(k)\right) \gamma^{*}=-\infty$. That is $\Delta_{\alpha(\ell)}^{2} v(k) \rightarrow-\infty$ for all large $k$. It follows that $\Delta_{\alpha(\ell)} v(k) \rightarrow-\infty, v(k) \rightarrow-\infty$, which contradicts the positivity of ${ }^{v(k)}$.

- If $\Delta_{\alpha(\ell)}\left(\Delta_{\alpha(\ell)}^{2} v(k)\right) \gamma^{*}>0$ eventually. Then combining this with $\Delta_{\alpha(\ell)}^{2}\left(\left(\Delta_{\alpha(\ell)}^{2} v(k)\right) \gamma^{*}\right)<0$, we see that $\Delta_{\alpha(\ell)}\left(\Delta_{\alpha(\ell)}^{2} v(k)\right)^{*} \rightarrow 0$ or $\rightarrow a>0$ so $\left(\Delta_{\alpha(\ell)}^{2} v(k)\right)^{\gamma^{*}}=\left(\Delta_{\alpha(\ell)}^{2} v(k)\right) \gamma^{*}+\sum_{K}^{k-1}\left(\Delta_{\alpha(\ell)}^{2} v(k)\right) r^{*}$

If $\left(\Delta_{\alpha(\ell)}^{2} v(k)\right) \gamma^{*}>0$ That is $\Delta_{\alpha(\ell)}^{2} v(k)>0$ is increasing and $\rightarrow C$ or $\infty$. It follows that ${ }_{\alpha(\ell)} v(k)>0$; If $\left(\Delta_{\alpha(\ell)}^{2} v(k)\right) \gamma^{*}<0$. That is $\Delta_{\alpha(\ell)}^{2} v(k)<0$ is increasing and $\rightarrow d$ or 0 . If $\Delta_{\alpha(\ell)} v(k)<0$, then $v(k) \rightarrow \infty$, it is impossible, so $\Delta_{\alpha(\ell)} v(k)>0$. This completes the proof of the lemma.

From Lemma 1, we know $v(k), \Delta_{\alpha(\ell)} v(k), \Delta_{\alpha(\ell)}^{2} v(k), \Delta_{\alpha(\ell)}\left(\Delta_{\alpha(\ell)}^{2} v(k)\right) \gamma^{*}$ tend to finite or infinite limits as $k \rightarrow \infty$. Let $\lim _{k \rightarrow \infty} \Delta_{\alpha(\ell)}^{i} v(k)=z(i), i=0,1,2$, and $\lim _{k \rightarrow \infty} \Delta_{\alpha(\ell)}\left(\Delta_{\alpha(\ell)}^{2} v(k)\right) \gamma^{*}=z(3)$

It is that $z(3)$ is a finite nonnegative number. One can easily show that:

If $v(k)$ satisfies I, then the set of its asymptotic values ${ }^{z(i)}$ falls into one of the following three cases:

$I_{1} . z(0)=z(1)=z(2)=\infty, z(3) \in(0, \infty)$

$I_{2} . z(0)=z(1)=z(2)=\infty, z(3)=0$;

$I_{3} . z(0)=z(1)=\infty, z(2) \in(0, \infty), z(3)=0$.

If $v(k)$ satisfies II, then the set of its asymptotic values ${ }^{z(i)}$ falls into one of the following three cases:

$$
\begin{aligned}
& I I_{1} . z(0)=\infty, z(1) \in(0, \infty), z(2)=z(3)=0 \\
& I I_{2} . z(0)=\infty, z(1)=z(2)=z(3)=0
\end{aligned}
$$




$$
I_{3} . z(0) \in(0, \infty), z(1)=z(2)=z(3)=0 \text {. }
$$

Equivalent expressions for these six classes of positive solutions of (2) are as follows:

$$
\begin{aligned}
& I_{1} \cdot \lim _{k \rightarrow \infty} \frac{v(k)}{k^{2+\frac{1}{\gamma}}}=\text { const }>0 \\
& I_{2} \cdot \lim _{k \rightarrow \infty} \frac{v(k)}{k^{2+\frac{1}{\gamma}}}=0, \lim _{k \rightarrow \infty} \frac{v(k)}{k^{2}}=\infty ; \\
& I_{3} \cdot \lim _{k \rightarrow \infty} \frac{v(k)}{k^{2}}=\text { const }>0 ; \\
& I I_{1} \cdot \lim _{k \rightarrow \infty} \frac{v(k)}{k}=\text { const }>0 ; \\
& I I_{2} \cdot \lim _{k \rightarrow \infty} \frac{v(k)}{k}=0, \lim _{k \rightarrow \infty} v(k)=\infty ; \\
& I I_{3} \cdot \lim _{k \rightarrow \infty} v(k)=\text { const }
\end{aligned}
$$

Let $v(k)$ be a positive solution of(2) such that $v(k)>0, v(\sigma(k))>0$ for $k \geq K>k_{0}$. summing (2) from $k$ to $\infty$ gives

$$
\Delta_{\alpha(\ell)}\left(\Delta_{\alpha(\ell)}^{2} v(k)\right)^{\gamma^{*}}=z(3)+\sum_{s=k}^{\infty} q(s)(v(\sigma(k)))^{\beta}, k \geq K
$$

If $v(k)$ is a solution of type $I_{i}(i=1,2,3)$, then sum (3) three times over $[K, k-1]$ to obtain

$$
v(k)=n_{0}+n_{1}(k-K)+\sum_{s=K}^{k-1}(k-s)\left[n(2)^{\gamma}+\sum_{r=k}^{s-1}\left(z(3)+\sum_{\tau=r}^{\infty} g(\tau)(v(\sigma(\tau)))^{\beta}\right)\right]^{\frac{1}{\gamma}}
$$

For $k \geq K$ where $n_{0}=v(K), n_{1}=\Delta_{\alpha(\ell)} v(K), n_{2}=\Delta_{\alpha(\ell)}^{2} v(K)$ are nonnegative constants. The equality (4) gives a representation for a solution $v(k)$ of type ${ }^{-I_{1}}$. A type ${ }^{-I_{2}}$ solution $v(k)$ of (2) is expressed by (4) with $z(3)=0$.

If ${ }^{v(k)}$ is a solution of type ${ }^{I_{3}}$, then, first summing (2) from $k$ to $\infty$ and then summing the resulting equation twice times over $[K, k-1]$ to obtain

$$
v(k)=n_{0}+n_{1}(k-K)+\sum_{s=K}^{k-1}(k-s)\left[z(2)^{\gamma}-\sum_{r=s}^{\infty}(r-s) q(r)(v(\sigma(r)))^{\beta}\right]^{\frac{1}{\gamma}}, k>K
$$

A representation for a solution $v(k)$ of type ${ }^{I I_{1}}$ is derived by summing (3) with $z(3)=0$ twice from $k$ to $\infty$ and then once from $K$ to $k-1$ : 
$v(k)=n_{0}+\sum_{s=K}^{k-1}\left(z(1)+\sum_{r=s}^{\infty}\left[\sum_{\tau=r}^{\infty}(\tau-r) q(\tau) v(\sigma(\tau))^{\beta}\right]\right)^{\frac{1}{\gamma}}, k>K$

A representation for a solution $v(k)$ of type $I_{2}$ is given by (6) with $z(3)=0$ a representation for a solution $v(k)$ of type $I_{3}$ is derived by summing (3) with $z(3)=0$ three times from $k$ to $\infty$ yield

$v(k)=z(0)-\sum_{s=k}^{\infty}(s-k)\left[\sum_{r=s}^{\infty}(r-s) q(r) v(\sigma(r))^{\beta}\right]^{\frac{1}{\gamma}}, k>K$

\section{NONOSCILLATION CRITERIA}

Theorem 1: The equation (2) has a positive of type- $I_{1}$ if and only if

$$
\sum_{k=k_{0}}^{\infty} q(k)(\sigma(k))^{2+\frac{1}{\gamma}} \beta<\infty
$$

Proof Necessary: Suppose that (2) has a positive of type- $I_{1}$, then, it satisfies (4) for $k \geq K$, which implies that

$$
\sum_{k=k_{0}}^{\infty} q(k)(v(\sigma(k)))^{\beta}<\infty
$$

This together with the asymptotic relation $\lim _{k \rightarrow \infty}=$ const $>0$; shows that the condition (8) is satisfied.

Sufficiently: Suppose now that (8) holds. Let $n>0$ be any given constant. Choose $K>k_{0}$ large enough so that

$$
\left(\frac{\gamma^{2}}{(\gamma+1)(2 \gamma+1)}\right)^{\beta} \sum_{k=k_{0}}^{\infty} q(k)(\sigma(k))^{2+\frac{1}{\gamma}} \beta \leq \frac{(2 n)^{\gamma}-n \gamma}{(2 n)^{\beta}}
$$

Put $K_{*} \min \left\{K, \inf _{k>K} \sigma(k)\right\}$, and define

$$
\begin{aligned}
& G(k, K)=\sum_{s=K}^{k-1}(k-s)(s-K)^{\frac{1}{\gamma}}=\frac{\gamma^{2}}{(\gamma+1)(2 \gamma+1)}(k-K)^{\frac{2}{1+\gamma}} k \geq K \\
& G(k, K)=0 \quad k<K
\end{aligned}
$$

Let $B(K)$ be the Banach space of all real sequences $V=v(k)$, with the norm $\|V\|=\sup _{k>k_{0}}|v(k)|<\infty \quad$ we define a closed, bounded and convex subset $\Omega$ of $B(K)$ as follows:

$$
\Omega=\left\{Y=v(k) \in B(K) n G(k, K) \leq v(k) \leq 2 n G(k, K), k \geq K_{*}\right\}
$$

Define the map $T: \Omega \rightarrow B(K)$ as follows: 


$$
\begin{cases}\operatorname{Tv}(k)=\sum_{K}^{k-1}(k-s)\left[\sum_{K}^{s-1}\left(n \gamma+\sum_{\tau=r}^{\infty} q(\tau)(v(\sigma(\tau)))^{\beta}\right)\right]^{\frac{1}{\gamma}}, & k \geq K \\ \operatorname{Tv}(k))=\operatorname{Tv}(K), & K_{*} \leq k \leq K\end{cases}
$$

I. $T$ Maps $\Omega$ into $\Omega$. For $v(k) \in \Omega$, then for $k \geq K$

$$
\operatorname{Tv}(k) \geq k \sum_{K}^{k-1}(k-s)(s-K)^{\frac{1}{\gamma}}=k G(k, K)
$$

And

$$
\begin{aligned}
& \operatorname{Tv}(k) \leq \sum_{K}^{k-1}(k-s)\left[\sum_{K}^{k-1}\left(n \gamma+\sum_{\tau=r}^{\infty} q(\tau)\left(2 n \sigma(\sigma(\tau), K)^{\beta}\right)\right)\right]^{\frac{1}{\gamma}} \\
& \leq \sum_{K}^{k-1}(k-s)\left[\sum_{K}^{k-1}\left(n \gamma+\left(\frac{2 n \gamma^{2}}{(\gamma+1)(2 \gamma+1)}\right)^{\beta}\right) \sum_{\tau=r}^{\infty} q(\tau)(\sigma(\tau))^{\left(2+\frac{1}{\gamma}\right) \beta}\right]^{\frac{1}{\gamma}} \\
& \leq 2 n \sum_{K}^{k-1}(k-s)(s-K)^{\frac{1}{\gamma}}=2 n G(k, K)
\end{aligned}
$$

II. $\quad T$ is continuous. Let $v^{(n)} \in \Omega$ such that $\lim _{n \rightarrow \infty}\left\|v^{(n)}-v\right\|=0$

$$
\left|\left(T v^{(n)}\right)_{k}-(T v)_{k}\right|=\sum_{K}^{k-1}(k-s)\left[\sum_{K}^{s-1}\left(n \gamma+\sum_{\tau=r}^{\infty} q(\tau)\left(v^{(n)}(\sigma(\tau))\right)^{\beta}\right)\right]^{\frac{1}{\gamma}}-\sum_{K}^{k-1}(k-s)\left[\sum_{K}^{s-1}\left(n \gamma+\sum_{\tau=r}^{\infty} q(\tau)(v(\sigma(\tau)))^{\beta}\right)\right]^{\frac{1}{\gamma}}
$$

By using Lebesgue's dominated convergence theorem, we can conclude that

$$
\lim _{n \rightarrow \infty}\left\|\left(T v^{(n)}\right)-(T v)\right\|=0
$$

III. $\quad T$ is uniformly-cauchy, $\forall k_{1}, k_{2}>K_{*}$

$$
\begin{aligned}
& \left|\left(T v^{(n)}\right)_{k_{1}}-(T v)_{k_{2}}\right|=\sum_{K}^{k_{2}-1}(k-s)\left[\sum_{K}^{s-1}\left(n \gamma+\sum_{\tau=r}^{\infty} q(\tau)\left(v^{(n)}(\sigma(\tau))\right)^{\beta}\right)\right]^{\frac{1}{\gamma}}-\sum_{K}^{k_{1}-1}(k-s)\left[\sum_{K}^{s-1}\left(n \gamma+\sum_{\tau=r}^{\infty} q(\tau)(v(\sigma(\tau)))^{\beta}\right)\right]^{\frac{1}{\gamma}} \\
& =\sum_{K}^{k_{2}-1}(k-s)\left[\sum_{K}^{s-1}\left(n \gamma+\sum_{\tau=r}^{\infty} q(\tau)\left(v^{(n)}(\sigma(\tau))\right)^{\beta}\right)\right]^{\frac{1}{\gamma}}
\end{aligned}
$$

Therefore, by the Schauder fixed point theorem, there exists a fixed $T v=v$, which satisfies (2). This completes the proof.

Theorem 2: The equation (2) has a positive of type- $\mathrm{I}_{3}$ if and only if 


$$
\sum_{k=k_{0}}^{\infty} k q(k)(\sigma(k))^{2 \beta}<\infty
$$

Proof Necessary: Suppose that (2) has a positive of type- $\mathrm{I}_{3}$, then, it satisfies (5) for $k \geq K$, which implies that

$$
\sum_{k=K}^{\infty}(k-K) q(k)(\sigma(k))^{\beta}<\infty
$$

This together with the asymptotic relation $\lim _{k \rightarrow \infty} \frac{v(k)}{k^{2}}=$ const $>0$; shows that the condition (9) is satisfied.

Sufficiently: Suppose now that (9) holds. Let $k>0$ be any given constant. Choose $K>k_{0}$ large enough so that

$$
\sum_{k=K}^{\infty} k q(k)(\sigma(k))^{2 \beta} \leq \frac{(2 n)^{\gamma}-n \gamma}{(n)^{\beta}}
$$

Put $K_{*}=\min \left\{K, \inf f_{k>K} \sigma(k)\right\}$, Let $B_{K}$ be the Banach space of all real sequences $Y=v(k)$, with the norm $\|V\|=\sup _{k>k_{0}}|v(k)|<\infty$ we define a closed, bounded and convex subset $\Omega$ of $B_{K}$ as follows:

$$
\Omega=\left\{V=v(k) \in B_{K} \frac{2}{n}(k-K)_{+}^{2} \leq v(k) \leq n(k-K)_{+}^{2}, k \geq K_{*}\right\}
$$

Where $k-K_{+}=k-K$ if $k \geq K$, and $k-K_{+}=0$ if $k \leq K$. Define the map $T: \Omega \rightarrow B_{K}$ as follows:

$$
\begin{cases}\operatorname{Tv}(k)=\sum_{K}^{k-1}(k-s)\left[2 n \gamma-\sum_{r=s}^{\infty}(r-s) q(\tau)(v(\sigma(\tau)))^{\beta}\right]^{\frac{1}{\gamma}}, & \\ \operatorname{Tv}(k))=\operatorname{Tv}(K), & K * \leq k \leq K\end{cases}
$$

The proof is similar to that of Theorem 1 and there exists an element $v$ such that $v=T v$, which is a type- $\mathrm{I}_{3}$ solution of (2) with the property that $\lim _{k \rightarrow \infty} \Delta_{2 \alpha(\ell)} v(k)=2 n>0$. This completes the proof.

Theorem 3: The equation (2) has a positive of type- $\mathrm{II}_{1}$ if and only if

$$
\sum_{k=K}^{\infty}\left[\sum_{s=k}^{\infty}(s-k) q(s)(\sigma(s))^{\beta}\right]^{\frac{1}{\gamma}}<\infty
$$

Proof: Necessary. Suppose that (2) has a positive of type- $\mathrm{II}_{1}$, then, it satisfies (6) for $k \geq K$, which implies that

$$
\sum_{k=K}^{\infty}(k-K) q(k)(v(\sigma(k)))^{\beta}<\infty
$$

This together with the asymptotic relation $\lim _{k \rightarrow \infty} \frac{v(k)}{k}=$ const $>0$; shows that the condition (13) is satisfied.

Sufficiently: Suppose now that (13) holds. Let $n>0$ be any given constant. Choose $K>k_{0}$ large enough so that 
$\sum_{k=K}^{\infty}\left[\sum_{s=k}^{\infty}(s-k) q(s)(\sigma(s))^{\beta}\right]^{\frac{1}{\gamma}}<2 \frac{-\beta}{\gamma} n^{1-\frac{\beta}{\gamma}}$

Put $^{K *}=\min \left\{K, \inf _{k>K} \sigma(k)\right\}$. Let $B_{K}$ be the Banach space of all real sequences $V=v(k)$, with the norm $\|V\|=\sum_{k>k_{0}}|v(k)|<\infty$ define a closed, bounded and convex subset $\Omega$ of $B_{K}$ as follows:

$\Omega=\left\{V=v(k) \in B_{K} n k \leq v(k) \leq 2 n k, k \geq K_{*}\right\}$

Define the map $T: \Omega \rightarrow B_{K}$ as follows:

$$
\begin{cases}\operatorname{Tv}(k)=n k+\sum_{K}^{k-1} \lim _{s}^{\infty}\left[\sum_{r}^{\infty}(\tau-r) q(\tau)(v(\sigma(\tau)))^{\beta}\right]^{\frac{1}{\gamma}}, & k \geq K \\ \operatorname{Tv}(k)=n k, & K_{*} \leq k \leq K\end{cases}
$$

The proof is similar to that of Theorem 1 and there exists an element $v$ such that $v=T v$, which is a type- $\mathrm{II}_{1}$ solution of (2) with the property that $\lim _{k \rightarrow \infty} \Delta_{\alpha(\ell)} v(k)=k>0$; This completes the proof.

Theorem 4: The equation (2) has a positive of type- $\mathrm{II}_{3}$ if and only if

$$
\sum_{k=k_{0}}^{\infty} k\left[\sum_{s=k}^{\infty}(s-k) q(s)\right]^{\frac{1}{\gamma}}<\infty
$$

Proof Necessary: Suppose that (2) has a positive of type- $\mathrm{II}_{3}$, then, it satisfies (7) for $k \geq K$, which implies that

$$
\sum_{k=K}^{\infty} k\left[\sum_{s=k}^{\infty}(s-k) q(s)(v(\sigma(s)))^{\beta}\right]^{\frac{1}{\gamma}}<\infty
$$

This together with the asymptotic relation $\lim _{k \rightarrow \infty}=$ const $>0$; shows that the condition (15) is satisfied.

Sufficiently: Suppose now that (15) holds. Let $n>0$ be any given constant. Choose $K>k_{0}$ large enough so that

$$
\sum_{k=K}^{\infty} k\left[\sum_{s=k}^{\infty}(s-k) q(s)(v(\sigma(s)))^{\beta}\right]^{\frac{1}{\gamma}}<\frac{1}{2} n^{1-\frac{\beta}{\gamma}}
$$

Put $K_{*}=\min \left\{K, \inf _{k>K} \sigma(k)\right\}$. Let $B_{K}$ be the Banach space of all real sequences $V=v(k)$, with the norm $\|V\|=\sum_{k>k_{0}}|v(k)|<\infty$, we define a closed, bounded and convex subset $\Omega$ of $B_{K}$ as follows:

$$
\Omega=\left\{V=v(k) \in B_{K} \frac{n}{2} \leq v(k) \leq n, k \geq K_{*}\right\}
$$

Define the map $T: \Omega \rightarrow B_{K}$ as follows: 


$$
\begin{cases}\operatorname{Tv}(k)=n-\sum_{k}^{\infty}(s-k)\left[\sum_{r=s}^{\infty}(r-s) q(r)(v(\sigma(r)))^{\beta}\right]^{\frac{1}{\gamma}}, & k \geq K \\ \operatorname{Tv}(k)=\operatorname{Tv}(K), & K_{*} \leq k \leq K\end{cases}
$$

The proof is similar to that of Theorem 1 and there exists an element $v$ such that $v=T v$, which is a type-II 1 solution of (2) with the property that $\lim _{k \rightarrow \infty} \Delta_{\alpha(\ell)} v(k)=k>0$; This completes the proof.

Theorem 5: The equation (2) has a positive of type- $\mathrm{I}_{2}$ if

$$
\sum_{k=k_{0}}^{\infty} q(k)(v(\sigma(k)))^{\left(2+\frac{1}{\gamma}\right) \beta} \leq \infty
$$

And

$$
\sum_{k=k_{0}}^{\infty} k q(k)(v(\sigma(k)))^{2 \beta}=\infty
$$

Proof: Suppose now that (19) holds. Choose $K>k_{0}$ large enough so that

$$
\sum_{k=K}^{\infty} q(k)(v(\sigma(k)))^{\left(2+\frac{1}{\gamma}\right) \beta} \leq \frac{1}{2 \gamma+1}\left(\frac{(\gamma+1)(2 \gamma+1)}{\gamma^{2}}\right)^{\gamma}
$$

Put $_{*}=\min \left\{K, \inf _{k>K} \sigma(k)\right\}$. Let $B_{K}$ be the Banach space of all real sequences $V=v(k)$, with the norm $\|V\|=\sum_{k>k_{0}}|v(k)|<\infty$, we define a closed, bounded and convex subset $\Omega$ of $B_{K}$ as follows:

$\Omega=\left\{V=v(k) \in B_{K} \frac{1}{2^{1+\frac{1}{\gamma}}}(k-K)_{+}^{2} \leq v(k) \leq n^{2+\frac{1}{\gamma}}, k \geq K_{*}\right\}$

Define the map $T: \Omega \rightarrow B_{K}$ as follows:

$$
\begin{cases}\operatorname{Tv}(k)=n-\sum_{K}^{k-1}(k-s)\left[\frac{1}{2} \sum_{\tau=r}^{\infty}(\tau) q(\tau)(v(\sigma(\tau)))^{\beta}\right]^{\frac{1}{\gamma}}, & k \geq K \\ \operatorname{Tv}(k)=0, & K_{*} \leq k \leq K\end{cases}
$$

The proof is similar to that of Theorem 1 and there exists an element $v$ such that $v=T v$, which is a type- $\mathrm{I}_{2}$ solution of (2). This completes the proof.

Theorem 6: The equation (2) has a positive of type- $\mathrm{II}_{2}$ if

$$
\sum_{k}^{\infty} k\left[\sum_{k}^{\infty}(s-k) q(s)(\sigma(s))^{\beta}\right]^{\frac{1}{\gamma}}<\infty
$$

And 


$$
\sum_{k=k_{0}}^{\infty} k\left[\sum_{k}^{\infty}(s-k) q(s)\right]^{\frac{1}{\gamma}}=\infty
$$

Proof: Suppose now that (23) holds. Choose $K>k_{0}$ large enough so that

$$
\sum_{k=K}^{\infty}\left[\sum_{k}^{\infty}(s-k) q(s)(v(\sigma(s)))^{\beta}\right]^{\frac{1}{\gamma}} \leq 2^{\frac{-\beta}{\gamma}} n^{1-\frac{\beta}{\gamma}}
$$

Put $K_{*}=\min \left\{K, \inf _{k>K} \sigma(k)\right\}$. Let $B_{K}$ be the Banach space of all real sequences $V=v(k)$, with the $\underset{\text { norm }}{\|V\|=\sum_{k>k_{0}}|v(k)|<\infty}$, we define a closed, bounded and convex subset $\Omega$ of $B_{K}$ as follows:

$$
\Omega=\left\{V=v(k) \in B_{K} n \leq v(k) \leq 2 n k, k \geq K_{*}\right\}
$$

Define the map $T: \Omega \rightarrow B_{K}$ as follows:

$$
\begin{cases}\operatorname{Tv}(k)=n+\sum_{K}^{k-1}(s-k) \sum_{s}^{\infty}\left[\sum_{\tau=r}^{\infty}(\tau-r) q(\tau)(v(\sigma(\tau)))^{\beta}\right]^{\frac{1}{\gamma},}, & k \geq K \\ \operatorname{Tv}(k)=\operatorname{Tv}(K), & K_{*} \leq k \leq K\end{cases}
$$

The proof is similar to that of Theorem 1 and there exists an element $v$ such that $v=T v$, which is a type- $\mathrm{II}_{2}$ solution of (2) with the property that $\lim _{k \rightarrow \infty} \Delta_{\alpha(\ell)} v(k)=k>0$; This completes the proof.

\section{CONCLUSIONS}

In this paper we derived oscillation and nonoscillation heorems for a class of fourth order quasilinear generalized $\alpha$ - difference equations.. Also, results are verified and proved with suitable example verified by using matlab programme.

\section{ACKNOWLEDGEMENT}

I wish to thank various people for their contribution to this paper; Dr. V. Chandrasekar and Dr. A. Benevatho Jaison, for their valuable technical support and help on this paper. Special thanks should be given to Dr. S K. Khadar Babu, my research supervisor for her professional guidance and valuable support and for his useful and constructive recommendations on this paper.

\section{REFERENCES}

1. Zhang, B. G., \& Zhou, Y. (2001). Oscillation of a kind of two-variable function equation. Computers and Mathematics with application, 42,369-378.

2. Zhang, B. G. \& Yang, B. (1999). Oscillation in higher order nonlinear difference equation. Chznese Ann. Math., 20, 71-80.

3. Zhang, B. G. \& Wong, H. (1996). The existence of oscillatory and nonoscillatory solution of neutral difference equation. Chinese J. Math., 24, 377-393.

4. Zhou, Y. (1999). Oscillation of neutral difference equations. Kyungpook Math. J., 39, 283-291. 
5. Zhou, Y. (2001). Oscillation of higher-order linear difference equations, advance of difference equations III. Special Issue of Computers Math. Applic., 42, 323-331.

6. Lalli, B. S. \& Zhang, B. G. (1992). On existence of positive solutions and bounded oscillations for neutral difference equations. J. Math. Anal. Appl., 166, 272-287.

7. Zhang, B. G. \& Zhou, Y. (2000). Oscillation and nonoscillation of second order linear difference equation. Computers Math. Application, 39 (1/2), 1-7.

8. Erbe, L. \& Peterson, A. (2002). Oscillation criteria for second order matrix dynamic equations on a time scale. In R. P. Agarwal, M. Bohner, and D. O’Regan (Eds.), Special Issue on Dynamic Equations on Time Scales. J. Comput. Appl. Math., 141, 169-185.

9. Erbe, L. \& Peterson, A. (2004). Boundedness and oscillation for nonlinear dynamic equations on a time scale. Proc. Amer. Math. Soc., 132, 735-744.

10. Erbe, L. \& Peterson, A. \& Saker, S. H. (2003). Oscillation criteria for second-order nonlinear dynamic equations on time scales. J.London Math. Soc., 67, 701-714.

11. Hilger, S. (1990). Analysis on measure chains-a unified approach to continuous and discrete calculus. Results Math., 18, 1856.

12. Saker, S. H. (2004). Oscillation of nonlinear dynamic equations on time scales. Appl. Math. Comp., 148, 81-91.

13. Saker, S. H. (2005). Oscillation criteria of second-order half-linear dynamic equations on time scales, J. Comp. Appl. Math., $177,375-387$.

14. Ketab, Sattar, and Hussain Ali Mohamad. "Oscillation and Nonoscillation Properties of Solutions of Third Order Linear Neutral." International Journal of Applied Mathematics \& Statistical Sciences (IJAMSS) 5.3 (2016):31-38

15. Jawahar, G. Gomathi. "Qualitative Analysis on Second Order Neutral Delay Difference Equations." International Journal of Mechanical and Production Engineering Research and Development (IJMPERD) 9.2 (2019):659-664

16. Mohamad, H. A., and Sattar Naser Ketab. "Oscillation and nonoscillation properties of solutions of third order linear neutral differential equations." International Journal of Applied Mathematics \& Statistical Sciences (IJAMSS) 5.3 (2016): 31-38.

17. El-Sheikh, M. M. A., R. Sallam, and N. Mohamady. "New oscillation criteria for general neutral delay third order differential equations." International Journal of Mathematics and Computer Applications Research (IJMCAR) 3.2 (2013): 183-190. 